\title{
Prevalência de Enteroparasitos em Idosos
}

\author{
Prevalence of EtteroparasitesintheEderly
}

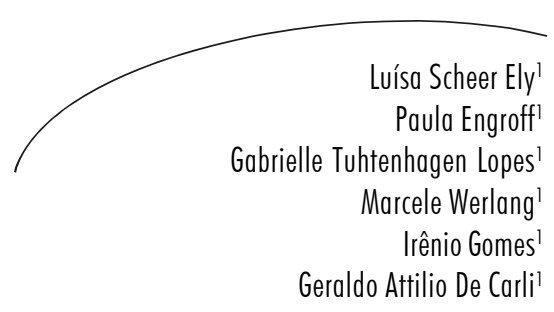

Resumo

Objetivos: Avaliar a prevalência de parasitos intestinais em idosos que frequentam um ambulatório geriátrico de Porto Alegre, no Rio Grande do Sul (RS), e moradores de duas instituições de longa permanência (ILP) do mesmo município. Analisar a presença de enteroparasitos nos animais de estimação desses idosos. Metodologia: $\mathrm{O}$ exame parasitológico das fezes (EPF) foi feito para diagnosticar a presença de parasitos nas fezes dos idosos e dos animais de estimação. Os métodos utilizados para a avaliação dos parasitos intestinais nas fezes foram o exame macroscópico, a técnica de sedimentação espontânea (técnica de Lutz ou Hoffman, Pons e Janer) e o método de Baermann-Moraes. As condições socioeconômicas e higiênicas dos pacientes foram avaliadas e, para aqueles que possuíam animal de estimação, foi aplicado um inquérito com os hábitos de higiene dos animais domésticos. Resultados: Trezentos e dez idosos foram estudados, com idade média de 78,6 $\pm 8,4$ anos; $77,1 \%$ eram mulheres e $22,9 \%$ eram homens. A prevalência de enteroparasitos encontrada foi de $12,9 \%$ para os idosos que frequentavam o ambulatório geriátrico e $12,9 \%$ para os institucionalizados. Os resultados mostraram que não houve associação entre o animal parasitado e o seu dono, pois nenhum idoso que possuía animal de estimação parasitado apresentou positividade pelo EPF. Conclusão: A prevalência de enteroparasitos encontrada nos idosos estudados em Porto Alegre foi mais baixa em relação aos dados existentes. Esses resultados mostram que cada região geográfica tem suas peculiaridades socioeconômicas, ambientais e educacionais. Os inquéritos coproparasitológicos devem continuar sendo realizados nos idosos das diferentes regiões do RS e do Brasil para identificar situações particulares, com o objetivo de propor medidas sanitárias e educativas para melhorar o estado de saúde das populações idosas.

\footnotetext{
1 Instituto de Geriatria e Gerontologia. Pontifícia Universidade Católica do Rio Grande do Sul (PUCRS).
}

Palavras-chave: Doenças Parasitárias. Idoso. Prevalência. Saúde do idoso. 
Abstract

Objectives: To evaluate the prevalence of intestinal parasites in elderly patients from a geriatric ambulatory in Porto Alegre, Rio Grande do Sul state, Brazil, and in elderly residents of two long-stay institutions in the same city, as well as to evaluate the presence of enteroparasites in the elderly's pets. Methods: Stool samples from the elderly and the pets were analyzed through fecal parasitological examination. To evaluate the enteroparasites, macroscopic exam, concentration methods of Hoffman, Pons and Janer and the Baermann-Moraes method were used. Socioeconomic conditions and hygiene habits were evaluated, and for the elderly that had pets a questionnaire about their way of life was applied. Results: 310 elderly were studied with mean age of $78.6 \pm 8.4$ years old, $77.1 \%$ were women and $22.9 \%$ were man. The prevalence of intestinal parasites was $12.9 \%$ for the elderly from the geriatric ambulatory and $12.9 \%$ for the institutionalized elderly. The results showed no association between enteroparasites from pets and their owner, because no elder who had a pet with parasitic disease presented positive result in fecal examination. Conclusions: The prevalence of enteroparasites in the elderly from Porto Alegre was lower than in other studies. These results showed that each geographic area has its socioeconomic, environmental, and educational peculiarities. The studies should be continued in the elderly from different areas of Rio Grande do Sul and Brazil to identify particular situations. Sanitary measures and education can be proposed based on such studies to improve population health.
Key words: Parasitic Diseases. Aged. Prevalence. Health of the Elderly.

\section{INTRODUÇÃO}

O último relatório estatístico da Organização Mundial da Saúde (OMS) sobre a saúde no mundo indicou que o Brasil apresentou melhora sensível em seus indicadores de saúde entre 1990 e 2007. Esses indicadores mostraram que a expectativa de vida dos brasileiros passou de 66 para 73 anos, e a mortalidade infantil $(<5$ anos de idade) caiu de 58/1.000 nascidos vivos para 22/ 1.000 nascidos vivos, apontando para um incremento na população de idosos. ${ }^{1} \mathrm{~A}$ expectativa de vida dos brasileiros, que em 1900 não alcançava os 35 anos de idade, atingiu 43 anos em 1950 e 68 anos em 2000, com a expectativa de atingir os 80 anos em 2025. ${ }^{2}$ A proporção de idosos, entre 1998 e 2008 , aumentou de $8,8 \%$ para $11,1 \%$. Os estados do Rio de Janeiro (14,9\%) e Rio Grande do Sul (RS) $(13,5 \%)$ continuam sendo as regiões com o maior número de idosos. ${ }^{3} \mathrm{Em} 1998$ eram, junto com a Paraíba, os únicos estados em que os idosos representavam mais de $10,0 \%$ da população. Atualmente, todos os estados do Sudeste e do Sul, assim como a maioria do Nordeste, já alcançaram essa proporção. ${ }^{4}$
O idoso, na maioria das vezes, apresenta um aumento na suscetibilidade ao desenvolvimento de câncer, doenças autoimunes e infecciosas. Em comparação a indivíduos jovens, os idosos apresentam diversos eventos que os predispõem a infecções, condições que aumentam a morbidade e mortalidade. ${ }^{5}$ Essa alta suscetibilidade dos idosos está associada à diminuição das funções normais do sistema imunológico. A população de linfócitos $\mathrm{T}$ é alterada com o avanço da idade, portanto a eficiência dos monócitos/macrófagos em destruir invasores fica prejudicada. ${ }^{6.8} \mathrm{Os}$ idosos residentes nas instituições de longa permanência (ILPs) apresentam maior grau de dependência e de doenças clínicas do que os idosos que vivem na comunidade, apresentando maior risco de adquirir doenças infecciosas. As infecções respiratórias, gastrointestinais, do trato urinário e da pele estão entre as infecções mais comuns nos residentes das ILPs. ${ }^{5}$

Os enteroparasitos contribuem para o comprometimento do estado nutricional dos idosos e da sua saúde. Os idosos geralmente desenvolvem diversas tarefas domésticas no seu 
dia a dia, tais como cultivo de hortas caseiras e limpeza do quintal, que podem favorecer a contaminação. ${ }^{9}$ Os parasitos intestinais podem causar a seus portadores obstrução intestinal, desnutrição, anemia ferropriva, diarreia e má absorção. ${ }^{10,11}$

A transmissão dos parasitos intestinais normalmente ocorre pela contaminação oral por cistos e ovos, pela água e pelos alimentos, e por mãos contaminadas com resíduos fecais de humanos e/ou de animais. ${ }^{10-13}$ Com o aumento do número de animais de estimação nos centros urbanos, a exposição humana aos agentes zoonóticos também aumentou. Embora os cães e gatos sejam, muitas vezes, considerados "membros da família”, é importante enfatizar que eles podem ser vetores de infecções por enteroparasitos. ${ }^{13}$

As campanhas de controle e erradicação das enteroparasitoses não requerem grandes financiamentos, capacidade humana e complexo equipamento médico de alta tecnologia, mas de uma imediata conscientização das populações sobre higiene e educação sanitária, e de uma urgente campanha de tratamento em massa. ${ }^{14,15}$

Devido à não existência de dados relatando as enteroparasitoses nos idosos no estado do RS, o objetivo deste estudo foi relatar a prevalência de enteroparasitos em idosos que frequentam um ambulatório geriátrico do município de Porto Alegre - RS, e em idosos moradores de duas ILPs do mesmo município, bem como avaliar a presença de enteroparasitos nos animais de estimação (cães e gatos) dos idosos que frequentam o ambulatório geriátrico.

\section{METODOLOGIA}

Esta pesquisa é um estudo transversal. A população foi formada por idosos que frequentaram um ambulatório geriátrico do município de Porto Alegre - RS, durante o período de setembro de 2009 a novembro de 2010, e por idosos moradores de duas ILPs do mesmo município durante o período de setembro de 2009 a maio de 2010. Os idosos que frequentavam o ambulatório geriátrico foram atendidos pelo Sistema Único de Saúde (SUS) ou por convênios. As ILPs estudadas abrigam idosos carentes, sendo que aproximadamente $40 \%$ não possuem vínculo familiar.

Os critérios de inclusão do estudo foram os idosos terem idade igual ou superior a 60 anos e serem moradores das duas ILPs estudadas, ou frequentarem o ambulatório geriátrico estudado. O critério de exclusão utilizado foi não possuírem condições de responder ao questionário e de colher a amostra das fezes, e de não terem auxílio para a colheita.

\section{Colheita das amostras}

Durante as visitas ao ambulatório geriátrico e às ILPs, os idosos receberam informações sobre o propósito do estudo e sobre os problemas de higiene e educação sanitária. Em seguida, houve a obtenção da assinatura do termo de consentimento livre e esclarecido (TCLE), e foi aplicado ao idoso um questionário com dados socioeconômicos e de higiene, além do questionário de inquérito sobre animais domésticos para os idosos do ambulatório geriátrico que possuíam animais de estimação. Foram elaborados dois questionários distintos, levando em consideração o estilo de vida diferenciado entre os idosos institucionalizados e os que viviam em suas residências.

Cada idoso recebeu um pote plástico limpo e seco, com tampa de rosca, para a colheita das fezes humanas e dos animais. Instruções impressas foram entregues para que a colheita das fezes fosse feita corretamente. Colheu-se uma amostra de fezes de cada participante do estudo e de cada animal de estimação. Exames parasitológicos das fezes (EPF) positivos foram encaminhados para orientação de médicos ou de veterinários; após o tratamento, realizou-se um novo EPF.

\section{Métodos de exame}

A presença de enteroparasitos foi diagnosticada nas fezes dos idosos e dos animais de estimação 
dos pacientes que frequentavam o ambulatório geriátrico. Os métodos utilizados para a avaliação dos parasitos intestinais nas fezes foram o exame macroscópico, para verificar a presença de vermes adultos, a técnica de sedimentação espontânea (técnica de Lutz ou Hoffman, Pons e Janer), ${ }^{16,17}$ para diagnosticar a presença de larvas, cistos e ovos, e o método de Baermann-Moraes, ${ }^{16,18}$ para isolamento de larvas nematóides.

\section{Considerações éticas}

O presente estudo foi aprovado pelo Comitê de Ética em Pesquisa da Pontifícia Universidade Católica do Rio Grande do Sul (PUCRS) sob o protocolo $\mathrm{n}^{\mathrm{o}}$. 09/04738. Todos os pacientes participantes do estudo assinaram o TCLE.

\section{Análise estatística}

O banco de dados foi estruturado em Excel e, posteriormente, analisado no SPSS versão 17.0. Foi calculada a prevalência em cada população com respectivo intervalo de confiança (IC) de $95 \%$; a associação entre as prevalências de enteroparasitos em animais e em idosos associadas com os dados socioeconômicos e higiênicos foram testadas pelo teste qui-quadrado. Foram considerados estatisticamente significativos os valores de $P<0,05$.

\section{RESULTADOS}

Foram estudados 310 idosos com média de idade de 78,6 $\pm 8,4$ anos; $77,1 \%$ eram do sexo feminino e 22,9\%, do sexo masculino. A prevalência de enteroparasitos diagnosticada foi de 12,9\%. Entre os pacientes da pesquisa, 163 frequentavam um ambulatório geriátrico de Porto Alegre e 147 eram provenientes de duas ILPs do mesmo município. A média de idade dos idosos do ambulatório geriátrico foi de $76 \pm 7,2$ anos, e dos moradores das duas ILPs, foi de $73 \pm 11,3$ anos. A prevalência de enteroparasitos no ambulatório geriátrico e nas ILPs foi de 12,9\% (tabela 1).

Tabela 1 - Prevalência de enteroparasitos nos idosos do ambulatório geriátrico e nos moradores das ILPs, no período de setembro/2009 a novembro/2010, Porto Alegre, RS.

\begin{tabular}{lcc}
\hline \multicolumn{1}{c}{ EPF $^{\S}$} & $\mathrm{N}(\%)$ & $\mathrm{IC} * 95 \%$ \\
\hline Ambulatório Geriátrico & & \\
Amostras Negativas & $142(87,1)$ & $81,0 \%-91,8 \%$ \\
Amostras Positivas & $21(12,9)$ & $8,2 \%-19,0 \%$ \\
ILP $^{*}$ & & \\
Amostras Negativas & $128(87,1)$ & $80,6 \%-92,0 \%$ \\
Amostras Positivas & $19(12,9)$ & $8,0 \%-19,4 \%$ \\
\hline TOTAL & $270(87,1)$ & $82,8 \%-90,6 \%$ \\
Amostras Negativas & $40(12,9)$ & $9,5 \%-17,3 \%$ \\
Amostras Positivas &
\end{tabular}

${ }^{\mathrm{S}} \mathrm{EPF}=$ Exame Parasitológico das Fezes; *IC = Intervalo de Confiança;

"ILP = Instituições de Longa Permanência

A tabela 2 identifica a prevalência dos enteroparasitos associada aos dados demográficos e de higiene dos idosos estudados. Essa tabela possui alguns resultados que são referentes somente ao questionário feito para os idosos do ambulatório geriátrico, pois estas questões não se aplicam à realidade dos idosos moradores de ILPs. 
Tabela 2 - Prevalência de enteroparasitos, dados demográficos e de higiene dos idosos do ambulatório geriátrico e das duas ILPs de setembro/2009 a maio/2010, Porto Alegre, RS.

\begin{tabular}{|c|c|c|c|}
\hline & População N (\%) & $\begin{array}{c}\text { Prevalência de } \\
\text { enteroparasitos \% }\end{array}$ & $P$ \\
\hline \multicolumn{4}{|l|}{ Sexo } \\
\hline Feminino & $239(77,1)$ & 12,6 & \multirow[t]{2}{*}{0,735} \\
\hline Masculino & $71(22,9)$ & 14,1 & \\
\hline \multicolumn{4}{|l|}{ Idade } \\
\hline 60-69 anos & $46(14,8)$ & 15,2 & \multirow{3}{*}{$0,029^{\#}$} \\
\hline 70-79 anos & $127(41,0)$ & 18,1 & \\
\hline 80 ou mais & $137(44,2)$ & 7,3 & \\
\hline \multicolumn{4}{|l|}{ Escolaridade ${ }^{¥}$} \\
\hline Até Ensino Fundamental & $156(83,4)$ & 14,7 & \multirow[t]{2}{*}{0,099} \\
\hline Ensino Médio ou mais & & 3,7 & \\
\hline \multicolumn{4}{|l|}{ Moradia ${ }^{¥}$} \\
\hline Casa & $135(82,8)$ & 15,6 & \multirow[t]{2}{*}{$0,014^{\#}$} \\
\hline Apartamento & $28(17,2)$ & 0,0 & \\
\hline \multicolumn{4}{|l|}{ Alimentação ${ }^{¥}$} \\
\hline Verduras e frutas & $15(9,2)$ & 40,0 & \multirow{3}{*}{$0,011^{\#}$} \\
\hline Carnes, pães e massas & $5(3,1)$ & 0,0 & \\
\hline Tudo & $143(87,7)$ & 10,5 & \\
\hline \multicolumn{4}{|c|}{ Costuma lavar frutas e verduras } \\
\hline Sim, com água & $138(84,7)$ & 13,0 & \multirow[t]{2}{*}{0,593} \\
\hline Sim, com água sanitária & $25(15,3)$ & 12,0 & \\
\hline \multicolumn{4}{|l|}{ Trabalha no jardim/horta } \\
\hline Sim & $88(28,4)$ & 13,6 & \multirow[t]{2}{*}{0,808} \\
\hline Não & $222(71,6)$ & 12,6 & \\
\hline \multicolumn{4}{|c|}{ Lava as mãos antes das refeições } \\
\hline $\operatorname{Sim}$ & $257(82,9)$ & 12,5 & \multirow[t]{2}{*}{0,601} \\
\hline Não & $53(17,1)$ & 15,1 & \\
\hline \multicolumn{4}{|l|}{ Lava as mãos após defecar } \\
\hline $\operatorname{Sim}$ & $264(85,2)$ & $\begin{array}{l}11,4 \\
217\end{array}$ & \multirow[t]{2}{*}{$0,050^{*}$} \\
\hline Não & $46(14,8)$ & & \\
\hline \multicolumn{4}{|l|}{ Renda familiar ${ }^{¥}$} \\
\hline Até $1 \mathrm{SM}^{*}$ & $13(8,0)$ & 23,1 & \multirow{4}{*}{0,417} \\
\hline $1-2 \mathrm{SM}$ & $94(57,7)$ & 11,7 & \\
\hline $2-3 \mathrm{SM}$ & $50(30,7)$ & 14,0 & \\
\hline Mais de 4 SM & $6(3,7)$ & 0,0 & \\
\hline \multicolumn{4}{|l|}{ Limpeza da região anal $\sqrt{ }$} \\
\hline Papel higiênico & $105(71,4)$ & 11,4 & \multirow{3}{*}{0,375} \\
\hline Água & $40(27,2)$ & 15,0 & \\
\hline Não faz & $2(1,4)$ & 50,0 & \\
\hline \multicolumn{4}{|l|}{ Possui coceira na região anal $\mathbb{}$} \\
\hline $\operatorname{Sim}$ & $19(12,9)$ & 5,3 & 0,255 \\
\hline Não & $128(87,1)$ & 14,1 & \\
\hline Realizou $\mathrm{EPF}^{\S}$ anteriormen & & & \\
\hline $\operatorname{Sim}$ & $228(73,5)$ & 13,6 & 0,544 \\
\hline Não & $82(26,5)$ & 11,0 & \\
\hline Tomou antiparasitário anter & & & \\
\hline $\operatorname{Sim}$ & $162(52,3)$ & 13,0 & 0,974 \\
\hline Não & $148(47,7)$ & 12,8 & \\
\hline
\end{tabular}

* SM = Salário Mínimo; ${ }^{\circledR}$ EPF $=$ Exame Parasitológico das Fezes; ${ }^{\# P}<0,05 ;{ }^{*}$ Dados somente do Ambulatório Geriátrico; ${ }^{£}$ Dados somente das ILPs. ILPs = Instituições de Longa Permanência. 
Os resultados mostraram que não houve associação entre o animal parasitado e o seu dono, pois nenhum idoso que possuía animal de estimação parasitado apresentou positividade no
EPF (tabela 3). Nem todos os idosos que tinham animal de estimação trouxeram as fezes dos animais para a realização do EPF, portanto o exame foi feito nas fezes de 39 animais (cães e gatos).

Tabela 3 - Prevalência de enteroparasitos em 65 idosos do ambulatório geriátrico que possuíam animal de estimação em relação aos hábitos dos animais. Porto Alegre, RS, de setembro/2009 a novembro/ 2010.

\begin{tabular}{|c|c|c|c|}
\hline & $\begin{array}{l}\text { População } \\
\text { N (\%) }\end{array}$ & $\begin{array}{c}\text { Prevalência de } \\
\text { enteroparasitos nos } \\
\text { idosos \% }\end{array}$ & $P$ \\
\hline \multicolumn{4}{|c|}{ Possui animal de estimação } \\
\hline $\operatorname{Sim}$ & $65(39,9)$ & 12,3 & \multirow[t]{2}{*}{0,858} \\
\hline Não & $98(60,1)$ & 13,3 & \\
\hline \multicolumn{4}{|c|}{ Possui animal de estimação parasitado } \\
\hline $\operatorname{Sim}$ & $5(3,1)$ & 0,0 & \multirow[t]{2}{*}{0,497} \\
\hline Não & $158(96,9)$ & 13,3 & \\
\hline \multicolumn{4}{|l|}{ Animal } \\
\hline Gato & $15(23,1)$ & 6,7 & \multirow[t]{2}{*}{0,403} \\
\hline Cachorro & $50(76,9)$ & 14,0 & \\
\hline \multicolumn{4}{|l|}{ Onde vive o animal } \\
\hline Dentro do domicílio & $20(30,8)$ & 5,0 & \multirow[t]{2}{*}{0,223} \\
\hline No pátio (rua) & $45(69,2)$ & 15,6 & \\
\hline \multicolumn{4}{|l|}{ Frequenta a cozinha } \\
\hline $\operatorname{Sim}$ & $44(67,7)$ & 11,4 & \multirow[t]{2}{*}{0,512} \\
\hline Não & $21(32,3)$ & 14,3 & \\
\hline \multicolumn{4}{|l|}{ Vacinado } \\
\hline $\operatorname{Sim}$ & $46(70,8)$ & 8,7 & \multirow[t]{2}{*}{0,166} \\
\hline Não & $19(29,2)$ & 21,1 & \\
\hline \multicolumn{4}{|l|}{ Toma vermífugo } \\
\hline $\operatorname{Sim}$ & $49(75,4)$ & 10,2 & \multirow[t]{2}{*}{0,306} \\
\hline Não & $16(24,6)$ & 18,8 & \\
\hline \multicolumn{4}{|l|}{ Onde costuma defecar } \\
\hline Cozinha & $4(6,2)$ & 0,0 & \multirow{4}{*}{0,680} \\
\hline Banheiro & $12(18,5)$ & 16,7 & \\
\hline Área de serviço & $48(73,8)$ & 12,5 & \\
\hline Rua & $1(1,5)$ & 0,0 & \\
\hline
\end{tabular}

Os parasitos não-patogênicos foram os mais frequentes nos idosos, 47,9\% para Entamoeba coli e
29,2\% para Endolimax nana. O parasito intestinal mais prevalente nos cães foi o Trichuris spp (tabela 4). 
Tabela 4 - Enteroparasitos encontrados em idosos moradores das duas ILPs, em pacientes do ambulatório geriátrico e em animais de estimação dos idosos. Porto Alegre, RS, período de setembro/2009 a novembro/2010, Porto Alegre, RS.

\begin{tabular}{lcccc}
\hline \multicolumn{1}{c}{ Enteroparasitos } & $\begin{array}{c}\text { ILPs\# } \\
\text { N (\%) }\end{array}$ & $\begin{array}{c}\text { Ambulatório } \\
\text { Geriátrico } \\
\mathbf{N ~ ( \% ) *}\end{array}$ & $\begin{array}{c}\text { Animais de } \\
\text { Estimação } \\
\mathbf{N ~ ( \% ) * ~}\end{array}$ & $\begin{array}{c}\text { Total } \\
\mathbf{N}(\%)\end{array}$ \\
\hline Entamoeba coli & $9(47,5)$ & $14(60,9)$ & - & $23(47,9)$ \\
Endolimax nana & $7(36,9)$ & $7(30,4)$ & - & $14(29,2)$ \\
Entamoeba histolytica/ E. díspar*** & - & $2(8,7)$ & - & $2(4,2)$ \\
Trichuris trichiura & $1(5,2)$ & - & - & $1(2,1)$ \\
Enterobius vermicularis & $1(5,2)$ & - & - & $1(2,1)$ \\
Strongyloides stercoralis & $1(5,2)$ & - & - & $1(2,1)$ \\
Ancilostomídeos & - & - & $4(16,7)$ & $1(2,1)$ \\
Trichuris spp. & - & - & $1(16,7)$ & $1(2,1)$ \\
Taenia spp. & - & - & $\mathbf{6 ( 1 0 0 )}$ & $\mathbf{4 8}(\mathbf{1 0 0})$ \\
\hline \multicolumn{1}{c}{ Total N (\%) } & $\mathbf{1 9 ( 1 0 0 )}$ & $\mathbf{2 3 ( 1 0 0 )}$ & & \\
\hline
\end{tabular}

* Mais de um parasito por pessoa ou animal de estimação.

** O diagnóstico laboratorial diferencial entre Entamoeba histolytica e E. dispar não pode ser realizado tomando-se como base a morfologia, a não ser que sejam vistas hemácias ingeridas pelos trofozoítos (E. bistolytica).

${ }^{\text {"ILPs }}=$ Instituições de Longa Permanência

\section{DISCUSSÃO}

Devido à não-existência de dados sobre a prevalência de enteroparasitos em idosos no estado do RS, torna-se muito difícil estimar a repercussão do parasitismo intestinal sobre a saúde e a economia da população de idosos estudados em Porto Alegre. ${ }^{11,15}$

A prevalência de enteroparasitos encontradas nos idosos estudados em Porto Alegre foi de $12,9 \%$, tanto para os idosos institucionalizados quanto para os idosos que frequentavam o ambulatório geriátrico.

Essa taxa de positividade foi menor do que em outros estudos realizados no Brasil e na América Latina. Hurtado-Guerreiro et al. (2005) encontraram, em Nova Olinda, estado do Amazonas, uma prevalência de $72,8 \%$ de enteroparasitoses em 81 idosos. ${ }^{9}$ Sánchez et al. (1999) analisaram 161 idosos atendidos em um hospital da Costa Rica, onde encontraram uma prevalência de $26,5 \%$ da população parasitada por helmintos e protozoários. ${ }^{19}$ Chen et al. (1998) estudaram a prevalência de enteroparasitos em 493 idosos chilenos e encontraram $37,8 \%$ da população parasitada. ${ }^{20}$ Araújo e Correia (1997) estudaram 365 idosos de João Pessoa, no estado da Paraíba, e verificaram a percentagem de 55,1\% de parasitos intestinais. ${ }^{21}$ Esses resultados mostram que cada região geográfica tem suas peculiaridades socioeconômicas, ambientais e educacionais. É evidente, pois, que as populações que deram origem às amostras apresentam características diferentes, não oferecendo dados comparáveis. Com isso, sua significância se mostra limitada. ${ }^{11,15}$

Os resultados obtidos em nosso inquérito mostraram que os parasitos não-patogênicos representaram $77,1 \%$ dos casos positivos, ou seja, $47,9 \%$ para E. coli e $29,2 \%$ para E. nana. Outros estudos ${ }^{9,19,20,21}$ apresentam alta prevalência de $A$ scaris lumbricoides, Trichuris trichiura, Blastocystis hominis, Giardia lamblia e Entamoeba histolytica/E. dispar. Neves et al. (1995) e Ludwig et al. (1999) afirmam que, 
com o passar dos anos, há uma tendência para a diminuição progressiva nas taxas de prevalência de enteroparasitos em adultos, principalmente para $A$. lumbricoides, explicada pela mudança de hábitos de higiene e pelo desenvolvimento de uma imunidade progressiva de longa duração contra os parasitos. ${ }^{22,23}$

Esta pesquisa mostrou que a prevalência de enteroparasitos entre homens e mulheres foi semelhante tanto nos idosos institucionalizados quanto nos pacientes do ambulatório geriátrico. $O$ resultado pode ser observado em outros estudos, indicando que as infecções por enteroparasitos não dependem do sexo. ${ }^{24-27}$ Quanto à faixa etária, a pesquisa mostrou que os idosos acima de 80 anos apresentam menor taxa de infecção por enteroparasitos, provavelmente pelo fato de que muitos deles são dependentes e têm menos contato com o ambiente externo. ${ }^{2,3}$

Os idosos que relataram se alimentar de verduras e frutas tiveram um índice de infecção por enteroparasitos maior do que aqueles que se alimentam de carnes, pães e massas. Provavelmente esse fato explica a alta prevalência de enteroparasitos não-patogênicos, pois são considerados um indicador de alimentos e água contaminados por matéria fecal. ${ }^{28}$ Outro fato que pode explicar a maior prevalência dos parasitos não-patogênicos é o hábito de não lavar as mãos após defecar ou antes das refeições, reafirmando que o saneamento básico e a educação sanitária são as mais importantes medidas de higiene que devem ser implementadas. ${ }^{9} 14,15,19,28$

Os idosos que possuíam no máximo o ensino fundamental apresentaram índice de infecção de $14,7 \%$. Essa prevalência foi estatisticamente significativa quando comparada com os idosos que estudaram além do ensino fundamental. Esses dados reforçam que os maiores índices de infecções por enteroparasitos estão entre as populações com pouca educação e baixo nível socioeconômico, nas quais os padrões de vida, de higiene ambiental e de educação sanitária são inadequados e deficientes. ${ }^{11,14,15,19,21}$
Em relação à moradia, a positividade para parasitos intestinais foi encontrada apenas nos idosos que residiam em casas $(15,6 \%)$. As casas facilitam o contato com o ambiente externo, favorecendo o contato com o solo, que é um dos fatores importantes para a infecção humana através de geo-helmintos. ${ }^{29,30}$

Neste estudo, não foi encontrada associação entre a presença de parasitoses intestinais nos idosos e em seus animais de estimação. Os resultados mostraram que $12,3 \%$ dos idosos que possuíam animal de estimação estavam parasitados, enquanto $13,3 \%$ dos idosos que não possuíam cães e gatos também estavam parasitados.

A despeito dos baixos índices de infecção por enteroparasitos nos idosos que possuem animais domésticos deste estudo, deve-se levar em consideração que o cuidado com a saúde do animal de estimação e do homem inclui o controle das infecções parasitárias, especialmente nas crianças e nos idosos, que são as populações mais

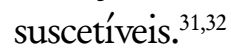

Visto que as infecções, mesmo quando moderadas, se tornam expressivas em hospedeiros imunodeprimidos ou com diminuição das funções normais do sistema imune, como os idosos, é essencial que os pacientes sejam examinados para se detectar a presença de parasitos através de exames repetidos, periódicos e/ou por diferentes procedimentos de diagnóstico. Os idosos mais vulneráveis sofrem não só os efeitos sobre seu estado imunológico, como também as repercussões sobre seu estado nutricional. ${ }^{6,7,8}$

As infecções por enteroparasitos podem ser controladas com eficácia, quando o estado socioeconômico de uma região e as condições sanitárias do meio ambiente são implementados e quando são implantadas outras medidas, como a educação para a saúde e a avaliação do estado nutricional da população. Tradicionalmente, a educação sanitária visa a promover os hábitos de higiene, favorecer uma nutrição adequada e criar condições ambientais condizentes ao sadio desenvolvimento físico e mental da população em geral. ${ }^{11,14,15,27,28}$ 


\section{CONCLUSÕES}

O estudo alcançou seus objetivos e demonstrou sua importância quanto à prevalência de parasitos intestinais na população idosa. Este inquérito provavelmente é o único que tem estudado as parasitoses intestinais em idosos no RS, pela inexistência de citações na literatura científica.

Não nos parece lícita uma comparação imediata entre os resultados apresentados pelos diferentes inquéritos no Brasile na América Latina, por terem sido feitos com amostras não padronizadas da população estudada e usando-se técnicas coprológicas de exame tampouco padronizadas.

\section{REFERÊNCIAS}

1. World Health Organization. World Health Statistics . Genebra: WHO;2009

2. Instituto Brasileiro de Geografia e Estatística Pesquisa(IBGE). Informação Demográfica e Socioeconômica. Síntese de Indicadores Sociais: uma análise das condições de vida da população brasileira. Rio de Janeiro: IBGE; 2009. 164-83p.

3. Chaimowics F. A saúde dos idosos brasileiros às vésperas século XXI: problemas, projeções e alternativas. Rev Saud Pública 1997; 31(2): 184 200.

4. Coelho Filho JF. Modelos de serviços hospitalares para casos agudos em idosos. Rev Saud Pública 2000; 31(6): 666-71.

5. Boás PJFV, Ferreira ALA. Infecção em idosos internados em instituição de longa permanência. Rev Associação Méd Brasileira 2007; 53(2): 126-9.

6. Wu D, Meydani SN. Age-associated changes in immune and inflammatory responses. J. Leukocyte Biol 2008; 84: 1-15.

7. Peres A, Nardi NB, Chies JAB. Imunossenescência: o envolvimento das Células T no Envelhecimento. Biociências 2003; 11(2): 187-94.

8. Albright JW, Albright JF. Ageing alters the competence of the immune system to control parasite infection. Immunology Letters 1994; 40(3): 279-85.

9. Hurtado-Guerrero AF, Alencar FH, HurtadoGuerrero JC. Ocorrência de enteroparasitos na população geronte de Nova Olinda do Norte :
$\mathrm{Na}$ prevenção das infecções parasitárias, é necessário identificar a fonte e o modo da infecção, que podem diferir em áreas geográficas e períodos sazonais. Entretanto, os inquéritos e as pesquisas devem continuar sendo realizados nos idosos das diferentes regiões do RS e do Brasil, para identificar situações particulares. O objetivo é propor medidas sanitárias e educacionais para melhorar o estado de saúde das populações.

Estudos de prevalência são necessários não só para se mensurar o problema das altas taxas de morbidade associadas a essas parasitoses, mas também para gerar dados para o planejamento de ações governamentais.

Amazonas, Brasil. Rev Acta Amazonica 2005; 35(4): 487-90.

10. Brandt LJ. Bloody Diarrhea in an Elderly Patient. Gatroenterology 2005; 126: 157-63.

11. Assis M, Borges FP, Santos RCV, et al. Prevalência de enteroparasitos em moradores de vilas periféricas de Porto Alegre. Rev Bras de Análises Clínicas 2003; 35(4): 215-7.

12. Guerrant RL, Gilder TV, Steiner ST, et al. Practice Guidelines for the Management of Infectious Diarrhea. Infectious Diseases Society of America Guidelines 2001; 32: 331-51.

13. Lourenço AEP, Uchoa CMA, Bastos OMP. Hospital food handlers in Niterói intestinal parasitism. Arq Latinoamericanos de Nutrición 2004; 54(4): 345-401.

14. De Carli GA, Tasca T, Machado ARL. Parasitoses Intestinais. In: Duncan BB, Schmidt MI, Giugliani ERJ. Medicina Ambulatorial: condutas de atenção primária baseadas em evidências. 3. ed. Porto Alegre: Artmed; 2004. 1465-75p.

15. Pavelecini RD, Borges FP, Michel RV, et al. Prevalência de infecções pelo Strongyloides stercoralis em uma área específica, vila dos papeleiros, na cidade de Porto alegre. Rev Bras Análises Clínicas 2004; 36(1): 19-21.

16. De Carli GA. Parasitologia Clínica: seleção de métodos e técnicas de laboratório para o diagnóstico de parasitoses em Humanos. 2. ed. São Paulo: Atheneu; 2008. 906p.

17. Hoffman WA, Pons JA, Janer SL. The concentration methods in schistosomiasis mansoni. J Public Health 1934; 9: 281-98. 
18. Baermann G. Eine einfache Methode zur Auffindung von Ankylostomun (Nematoden). In: Baermann G. Batavia: Neded Geneesk Labt Welteverden Feestbundel; 1917. 41-7p.

19. Sanchéz A, Mora J, Hernández F. Prevalencia de parasitos intestinales en adultos mayores, Hospital Raúl Bianco Cervantes. Rev Costarricense de Cienc Med 1999; 20(3-4): 167-73.

20. Chen SH, Codoceo A, Carrasco O, et al. Enteroparasitosis em La poblacion de La tercera edad consultante em centros médicos de La Pontificia Universidad Catolica de Chile. Parasitologia al día 1998; 22(3-4): 114-6.

21. Araújo CFF, Correia JS. Frequência de parasitoses intestinais em idosos dos núcleos da Prefeitura de João Pessoa. Rev Bras Análises Clínicas 1997; 29(4): 230-1.

22. Ludwig KM, Frei F, Alvares Filho F, et al. Correlação entre condições de saneamento básico e parasitoses intestinais na população de Assis. Rev Soc Bras Med Tropical 1999; 32(5): 547-55.

23. Neves DP, Melo AL, Genaro O, et al. Parasitologia Humana. 9. ed. São Paulo: Atheneu; 1995.524p.

24. De Carli GA, Mentz M, Rott MB, et al. Prevalência das enteroparasitoses na população urbana e rural da região carbonífera da cidade de Arroio dos Ratos.Rev Bras Análises Clínicas 1997; 78(4): 83-5.
25. Neves DP, Melo AL, Genaro O, et al. Parasitologia Humana. 9. ed. São Paulo: Atheneu; 1995.524p.

26. Ishiyama S, Rai SK, Ono K, et al. A small scale study on intestinal parasitosis in a remote village in Nepal. Nepal Medical College Journal 2003; 5: 28-30.

27. Rai SK, Matsumura T, Ono K, et al. Intestinal parasitosis in an "unknown disease outbreak" hit rural hilly area in western Nepal. Nepal Medical College Journal 2000; 2: 61-4.

28. Santos RCV, Hoerlle JL, Aquino ARC, et al. Prevalência de enteroparasitoses em pacientes ambulatoriais do Hospital Divina Providência de Porto Alegre. Rev Brás de Análises Clínicas 2004; 36(4): 241-3.

29. Rey L. Parasitologia. 4 ed. Rio de Janeiro: Guanabara Koogan; 2008. 930p.

30. Melo MCB, Klem VGQ, Mota JAC, et al. Parsitoses intestinais. Rev Méd 2004; 14: 3-12.

31. Lorenzini G, Tasca T, De Carli AG. Prevalence of intestinal parasites in dogs ans cats under veterinary care in Porto Alegre. J of Veterinary Research and Animal Science 2007; 44(2): 137-45.

32. Mentz MB, Rott MB, Jacobsen SIV, et al. Frequência de ovos de Toxocara spp. em três parques públicos da cidade de Porto Alegre. Rev Patologia Tropical 2004; 33(1): 105-12. 